\title{
SYSTEMS OF DIOPHANTINE EQUATIONS
}

\section{A. A. AUCOIN}

We first define the concept of equivalent solutions. Suppose $x_{k}$ $=\alpha_{k}, y_{i j}=\beta_{i j}$ is an integral solution of the system

$$
f_{i}\left(x_{1}, \cdots, x_{p}\right)=g_{i}\left(y_{i 1}, \cdots, y_{i q}\right) \quad(i=1, \cdots, n),
$$

where $f_{i}$ and $g_{i}$ are homogeneous polynomials with integral coeffcients, $f_{i}$ being of degree $n$ and $g_{i}$ being of degree $m$. If there are no integers $s>1, \alpha_{k}^{\prime}, \beta_{i j}^{\prime}$ such that $\alpha_{k}=s^{\lambda} \alpha_{k}^{\prime}, \beta_{i j}=s^{\mu} \beta_{i j}^{\prime}$, where $\lambda, \mu$ are positive integers such that $\lambda n=\mu m$, then $x_{k}=\alpha_{k}, y_{i j}=\beta_{i j}$ is defined to be a primitive solution of (1). If $x_{k}=\alpha_{k}, y_{i j}=\beta_{i j}$ is a primitive solution of (1), then $x_{k}=t^{\lambda} \alpha_{k}, y_{i j}=t^{\mu} \beta_{i j}$ (derived from the primitive solution), where $t \neq 0$ is an integer, $\lambda, \mu$ are any positive integers such that $\lambda n=\mu m$, is also a solution. Two solutions are said to be equivalent if they may be derived from the same primitive solution.

Our first theorem concerns the solution of the system ${ }^{1}$

$$
\prod_{j=1}^{n} \sum_{k=1}^{q} a_{i j k} x_{k}=f_{i}\left(y_{i}\right) \quad(i=1, \cdots, n),
$$

where $f_{i}\left(y_{i}\right)=f_{i}\left(y_{i 1}, \cdots, y_{i o}\right)$ are homogeneous polynomials of degree $m$, with integral coefficients, and $m$ and $n$ are relatively prime. We make the following preliminary definitions. $a_{i j k}$ are integers, $\lambda, \mu$ are positive integers such that $n \lambda=m \mu+1$. $p_{k}^{(h)}$ are integers such that $\sum_{k=1}^{q} a_{i j k} p_{k}^{(h)}=0(h=1, \cdots, n-1 ; j=1, \cdots, n-1 ; i=1, \cdots, n)$, $A_{i}=A_{i}(\alpha)=\prod_{j=1}^{n-1} \quad \sum_{k=1}^{a} a_{i j k} \alpha_{k}, p_{i n}=\sum_{k=1}^{q} a_{i n k} P_{k}^{(h)}, \quad p_{i n}=p_{i n}(\alpha)$ $=\sum_{k=1}^{q} a_{i n k} \alpha_{k}, A=A(\alpha)=\prod_{i=1}^{n} A_{i}, \bar{A}_{i}=A / A_{i}, P=P(\alpha)=\left|p_{i j}\right|$ is a determinant of order $n, P_{i j}$ is the cofactor of $p_{i j}$ in $P$, the $\alpha$ 's and $\beta$ 's being arbitrary integers.

THEOREM 1. Every integral solution $x_{k}, y_{i r}$ of (2) for which $P(x) \neq 0$ and $A(x) \neq 0$ is equivalent to a solution given by

$$
\begin{aligned}
& x_{k}=\sum_{h=1}^{n-1} p_{k}^{(h)} s_{h} t^{\lambda-1}+\alpha_{k} t^{\lambda} \quad(k=1, \cdots, q), \\
& y_{i r}=P A t^{\mu} \beta_{i r} \quad(i=1, \cdots, n ; r=1, \cdots, g),
\end{aligned}
$$

Presented to the Society, October 27, 1951; received by the editors July 17,1950 and, in revised form, April 21, 1951.

${ }^{1}$ Single equations of this type have been solved by two different methods. Sze A. A. Aucoin and W. V. Parker, Diophantine equations whose members are homogeneous, Bull. Amer. Math. Soc. vol. 45 (1939) pp. 330-331. See also A. A. Aucoin, Diophantine equations of degree n, Bull. Amer. Math. Soc. vol. 46 (1940) pp. 336-337. 
where

(4)

$$
\begin{aligned}
s_{h} & =P^{m-1} A^{m-1} \sum_{i=1}^{n} \bar{A}_{i} f_{i}\left(\beta_{i}\right) P_{i h} \quad(h=1, \cdots, n-1), \\
t & =P^{m-1} A^{m-1} \sum_{i=1}^{n} \bar{A}_{i} f_{i}\left(\beta_{i}\right) P_{i n} .
\end{aligned}
$$

Proof. If we let $x_{k}$ have the values given by (3), the left-hand member of (2) becomes

$$
\begin{aligned}
\prod_{j=1}^{n-1} \sum_{k=1}^{q} a_{i j k}\left[\sum_{h=1}^{n-1} p_{k}^{(h)} s_{h} t^{\lambda-1}+\alpha_{k} t^{\lambda}\right] \sum_{k=1}^{q} a_{i n k}\left[\sum_{h=1}^{n-1} p_{k}^{(h)} s_{h} t^{\lambda-1}+\alpha_{k} t^{\lambda}\right] \\
=\prod_{j=1}^{n-1}\left[t^{\lambda-1} \sum_{h=1}^{n-1} s_{h} \sum_{k=1}^{q} a_{i j k} p_{k}^{(h)}+t^{\lambda} \sum_{k=1}^{q} a_{i j k} \alpha_{k}\right] \\
\cdot t^{\lambda-1}\left[\sum_{h=1}^{n-1} s_{h} \sum_{k=1}^{q} a_{i n k} p_{k}^{(h)}+t \sum_{k=1}^{q} a_{i n k} \alpha_{k}\right] \\
=t^{n \lambda-1} \prod_{j=1}^{n-1} \sum_{k=1}^{q} a_{i j k} \alpha_{k}\left[\sum_{h=1}^{n-1} s_{h} p_{i h}+t p_{i n}\right] \\
=t^{n \lambda-1} A_{i}\left[\sum_{h=1}^{n-1} s_{h} p_{i h}+t p_{i n}\right] .
\end{aligned}
$$

Thus, if $x_{k}, y_{i r}$ have the values given by (3), (2) becomes, after the multiplication of each equation by the corresponding $\bar{A}_{i}$,

$$
t^{n \lambda-1} A\left[\sum_{h=1}^{n-1} s_{h} p_{i h}+t p_{i n}\right]=P^{m} A^{m} \bar{A}_{i} t^{m \mu} f_{i}\left(\beta_{i}\right) \quad(i=1, \cdots, n) .
$$

This system is identically satisfied in the $\alpha$ 's and $\beta$ 's if $s_{h}$ and $t$ are given by (4).

Suppose now that $x_{k}=\rho_{k}, y_{i r}=\nu_{i r}$ is any solution of (2). Then $\prod_{j=1}^{n} \sum_{i=1}^{q} a_{i j k} \rho_{k}=f_{i}\left(\nu_{i}\right)(i=1, \cdots, n)$. If we choose $\alpha_{k}=\rho_{k}, \beta_{i r}=\nu_{i r}$, we have

$$
\begin{aligned}
\bar{A}_{i} f_{i}\left(\beta_{i}\right) & =\bar{A}_{i} f_{i}\left(\nu_{i}\right) \\
& =\bar{A}_{i} \prod_{j=1}^{n} \sum_{k=1}^{q} a_{i j k \rho_{k}} \\
& =\bar{A}_{i} \prod_{j=1}^{n-1} \sum_{k=1}^{q} a_{i j k \rho_{k}} \sum_{k=1}^{q} a_{i n k \rho_{k}} \\
& =\bar{A}_{i} A_{i} p_{i n}=A p_{i n}
\end{aligned}
$$


from which it follows that $s_{h}=0(h=1, \cdots, n-1)$. Also

$$
t=P^{m-1} A^{m-1} \sum_{i=1}^{n} A p_{i n} P_{i n}=P^{m} A^{m} .
$$

Hence (3) becomes $x_{k}=\rho_{k} P^{m \lambda} A^{m \lambda}, y_{i r}=\nu_{i r} P^{m \mu+1} A^{m \mu+1}$ from which the theorem follows.

In the particular example

$$
\begin{aligned}
& (25 x-5 y-10 z+5 w)(29 x+2 y-5 z+10 w)(x+y+z+w)=p^{2}, \\
& (19 x+17 y+10 z+15 w)(4 x+7 y+5 z+5 w)(x+2 y+2 z+2 w)=q^{2}, \\
& (-29 x-2 y+5 z-10 w)(32 x-9 y-15 z+5 w)(x+3 y-2 z+2 w)=r^{2},
\end{aligned}
$$

the integers $p_{\boldsymbol{k}}^{(k)}$ are $(1,-2,3,-1)$ and $(1,3,-1,-4)$ and will make the first two factors of each equation on the left vanish.

The next system consists of two equations. Let $f_{1}(x)$ $=f_{1}\left(x_{1}, \cdots, x_{p}\right), f_{2}(x)=f_{2}\left(x_{1}, \cdots, x_{q}\right)$ be homogeneous polynomials of degree $n$ with integral coefficients. Suppose that integers $x_{i}=a_{i}$ exist such that all the partial derivatives of $f_{1}$, as well as those of $f_{2}$, of all orders less than $n-1$ vanish for $x_{i}=a$. Let $g_{1}(u)$ $=g_{1}\left(u_{1}, \cdots, u_{k}\right), g_{2}(v)=g_{2}\left(v_{1}, \cdots, v_{h}\right)$ be homogeneous polynomials ${ }^{2}$ with integral coefficients of degree $m$ where $m$ and $n$ are relatively prime. $\lambda$ and $\mu$ have the same meaning as in Theorem 1 .

THEOREM 2. Every integral solution of the system ${ }^{3}$

$$
f_{1}(x)=g_{1}(u), \quad f_{2}(x)=g_{2}(v)
$$

which does not satisfy

$$
A(x) f_{2}(x)-B(x) f_{1}(x)=0
$$

is equivalent to one given by

$$
x_{i}=a_{i} s^{\lambda-1}+\alpha_{i} \lambda^{\lambda}, \quad u_{i}=\beta_{j} R(\alpha) t^{\mu}, \quad v_{j}=\gamma_{j} R(\alpha) t^{\mu},
$$

where

$$
\begin{aligned}
A(\alpha) & =\sum_{j=1}^{p} a_{j} \frac{\partial f_{1}}{\partial \alpha_{j}}, \quad B(\alpha)=\sum_{j=1}^{q} a_{j} \frac{\partial f_{2}}{\partial \alpha_{j}}, \\
R(\alpha) & =A(\alpha) f_{2}(\alpha)-B(\alpha) f_{1}(\alpha), \\
s & =[R(\alpha)]^{m-1}\left[g_{1}(\beta) f_{2}(\alpha)-g_{2}(\gamma) f_{1}(\alpha)\right], \\
t & =[R(\alpha)]^{m-1}\left[A(\alpha) g_{2}(\gamma)-B(\alpha) g_{1}(\beta)\right],
\end{aligned}
$$

${ }^{2}$ We may assume that $g_{1}$ and $g_{2}$ are functions of the same variables. It is necessary that both $g_{1}$ and $g_{2}$ do not vanish identically.

${ }^{3}$ For single equations of this type see A. A. Aucoin, op. cit. pp. 334-335. 
the $\alpha$ 's, $\beta$ 's, and $\gamma$ 's being arbitrary integers.

PROOF. By Taylor's formula, if we let $x_{i}=a_{i} s t^{\lambda-1}+\alpha_{i} t^{\lambda}$,

$$
\begin{aligned}
& f_{1}(x)=s t^{n \lambda-1} \sum_{j=1}^{p} a_{j} \frac{\partial f_{1}}{\partial \alpha_{j}}+t^{n \lambda} f_{1}(\alpha), \\
& f_{2}(x)=s t^{n \lambda-1} \sum_{j=1}^{q} a_{j} \frac{\partial f_{2}}{\partial \alpha_{j}}+t^{n \lambda} f_{2}(\alpha) .
\end{aligned}
$$

Hence if $x_{i}, u_{j}, v_{j}$ have the values given by (7), (5) becomes

$$
\begin{aligned}
t^{n \lambda-1}\left[A(\alpha) s+f_{1}(\alpha) t\right] & =[R(\alpha)]^{m} t^{m \mu} g_{1}(\beta), \\
t^{n \lambda-1}\left[B(\alpha) s+f_{2}(\alpha) t\right] & =[R(\alpha)]^{m} t^{m \mu} g_{2}(\gamma),
\end{aligned}
$$

which is identically satisfied in the $\alpha^{\prime}$ 's, $\beta$ 's, and $\gamma^{\prime}$ 's if $s$ and $t$ are given by (8).

Suppose that $x_{i}=\rho_{i}, u_{j}=\delta_{j}, v_{j}=\nu_{j}$ is any given solution of (5). Then $f_{1}(\rho)=g_{1}(\delta), f_{2}(\rho)=g_{2}(\nu)$. If we choose $\alpha_{i}=\rho_{i}, \beta_{j}=\delta_{j}, \gamma_{j}=\nu_{j}$, then $s=0, t=[R(\rho)]^{m}$, and the solution becomes $x_{i}=\rho_{i}[R(\rho)]^{m \lambda}, u_{j}$ $=\delta_{j}[R(\rho)]^{m \mu+1}, v_{j}=\nu_{j}[R(\rho)]^{m \mu+1}$, which is equivalent to the given solution provided $R(\rho) \neq 0$, that is, provided $x_{i}=\rho_{i}$ does not satisfy (6).

One function which satisfies the conditions placed upon $f_{1}$ and $f_{2}$ is the determinant of order $n, D(x)=\left|a_{i j} x_{i j}\right|$ where the $a$ 's are integers and not all the $a$ 's in any row or column are zero. If there is one element $x_{p q}$ which occurs only once in $D(x)$, we may make the choice $x_{p q}=1, x_{i j}=0$ otherwise, and then all the partial derivatives of all orders less than $n-1$ vanish. It is not necessary, in some cases, that there be a unique element $x_{p q}$. If $a_{i j}=1$, for example, $D(x)$ may be the circulant. In this case the choice $x_{i j}=1$ is made.

Another function which satisfies the conditions imposed upon $f_{1}$ and $f_{2}$ is the function $P(x)=\prod_{i=1}^{n} \sum_{j=1}^{n} a_{i j} x_{j}$, where all the $a$ 's are integral and the determinant $\left|a_{i j}\right|$ does not vanish. For this function we may choose $x_{j}$ so that $n-1$ of the linear factors vanish and for this choice all the partial derivatives of $P(x)$ of all orders less than $n-1$ vanish.

As an example consider the equations

$$
\begin{aligned}
& x^{3}-x^{2} z-x y^{2}+y^{2} z=u^{2}, \\
& x^{3}-x^{2} y-x z^{2}+y z^{2}=v^{2} .
\end{aligned}
$$

The partial derivatives of the first order of the functions on the left vanish for $x=y=z=1$. We get, then, as solution $x=s+\alpha t, y=s+\beta t$, $z=s+\gamma t, u=D \lambda t, v=D \mu t$, where 


$$
\begin{aligned}
D & =2(\alpha-\beta)^{2}(\alpha-\gamma)^{2}(\gamma-\beta), \\
s & =D(\alpha-\beta)(\alpha-\gamma)\left[(\alpha+\gamma) \lambda^{2}-(\alpha+\beta) \mu^{2}\right], \\
t & =2 D(\alpha-\beta)(\alpha-\gamma)\left(\mu^{2}-\lambda^{2}\right) .
\end{aligned}
$$

If we choose $\alpha=3, \beta=2, \gamma=1, \lambda=-1, \mu=2$, we get as solution $x=-32, y=64, z=160, u=-768, v=1536$. It will be noted that $x, y, z$ have the factor $4^{2}$ while $u$ and $v$ have the factor $4^{3}$. Hence this solution is equivalent to the primitive solution $x=-2, y=4$, $z=10, u=-12, v=24$.

The third theorem treats a system for which there is no typical problem. The method will be illustrated by a particular system.

THEOREM 3. Every solution of the system

$$
\begin{aligned}
& f_{1}\left(x_{i}, y_{i}, z_{i}\right)=g_{1}\left(x_{i}, y_{i}, z_{i}\right), \\
& f_{2}\left(x_{i}, y_{i}, z_{i}\right)=g_{2}\left(x_{i}, y_{i}, z_{i}\right),
\end{aligned}
$$

for which the members do not vanish, where $f_{1}, f_{2}, g_{1}, g_{2}$ are homogeneous polynomials in each of the sets of variables, $f_{1}, f_{2}, g_{1}, g_{2}$ being of degrees $(4,6,2) ;(2,2,3) ;(7,1,1) ;(1,4,2)$ respectively in the variables $x_{i}, y_{i}, z_{i}$, is equivalent (in a sense to be defined) to a solution given by

$$
\begin{aligned}
& x_{i}=\alpha_{i} u^{7} v^{16} w^{19}, \\
& y_{i}=\beta_{i} u^{4} v^{9} w^{11}, \\
& z_{i}=\gamma_{i} u^{2} v w,
\end{aligned}
$$

where

$$
\begin{aligned}
u & =f_{1} f_{2} g_{1}^{2} g_{2}^{2}, \\
v & =f_{1} g_{2}, \\
w & =f_{2} g_{1},
\end{aligned}
$$

the $\alpha$ 's, $\beta$ 's, and $\gamma$ 's being arbitrary integers.

Proof. If we let $x_{i}, y_{i}, z_{i}$ have the values given by (10), then (9) becomes

$$
\begin{aligned}
u^{56} v^{120} w^{144} f_{1} & =u^{55} v^{122} w^{145} g_{1}, \\
u^{28} v^{53} w^{63} f_{2} & =u^{27} v^{54} w^{65} g_{2},
\end{aligned}
$$

and this system is satisfied identically in the $\alpha^{\prime} \mathrm{s}, \beta$ 's, and $\gamma^{\prime}$ s if $u, v, w$ are given by (11).

We now extend the concept of equivalent solutions. If $x_{i}=\alpha_{i}$, $y_{i}=\beta_{i}, z_{i}=\gamma_{i}$ is any solution of the system (9) and there are no integers $\alpha_{i}^{\prime}, \beta_{i}^{\prime}, \gamma_{i}^{\prime}$ and no positive integers $s, a, b$, and $c$ such 
that $\alpha_{i}=s^{a} \alpha_{i}^{\prime}, \beta_{i}=s^{b} \beta_{i}^{\prime}, \gamma_{i}=s^{c} \gamma_{i}^{\prime}$ where

$$
\begin{aligned}
& 4 a+6 b+2 c=7 a+b+c, \\
& 2 a+2 b+3 c=a+4 b+2 c,
\end{aligned}
$$

then $x_{i}=\alpha_{i}, y_{i}=\beta_{i}, z_{i}=\gamma_{i}$ is defined to be a primitive solution of (9). If $x_{i}=\alpha_{i}, y_{i}=\beta_{i}, z_{i}=\gamma_{i}$ is a primitive solution of (9), then $x_{i}=\alpha_{i} t^{a}$, $y_{i}=\beta_{i} t^{b}, z_{i}=\gamma_{i} t^{c}$ (derived from the primitive solution) where $t$ is a nonzero integer and $a, b, c$ are positive integers which satisfy (12), is also a solution. Two solutions are said to be equivalent if they may be derived from the same primitive solution.

Suppose now that $x_{i}=\lambda_{i}, y_{i}=\mu_{i}, z_{i}=\nu_{i}$ is any solution of (9). If we choose $\alpha_{i}=\lambda_{i}, \beta_{i}=\mu_{i}, \gamma_{i}=\nu_{i}$ we have that $x_{i}=\lambda_{i}\left(f_{1} f_{2}\right)^{56}, y_{i}=\mu_{i}\left(f_{1} f_{2}\right)^{32}$, $\boldsymbol{z}_{i}=\nu_{i}\left(f_{1} f_{2}\right)^{8}$, which is equivalent to the given solution.

The UNIVERSITY OF Houston 